\title{
Jordan frame beyond scalar-tensor theories
}

\author{
Fethi M. Ramazanoğlu ${ }^{1}$ \\ ${ }^{1}$ Department of Physics, Koç University, \\ Rumelifeneri Yolu, 34450 Sariyer, Istanbul, Turkey
}

(Dated: January 3, 2019)

\begin{abstract}
We study the Jordan frame formulation of generalizations of scalar-tensor theories conceived by replacing the scalar with other fields such as vectors. The generic theory in this family contains higher order time derivative terms in the Jordan frame action which is indicative of ill-posedness. However, we show that equations of motion can always be reduced to a second-order-in-time form as long as the original Einstein frame formulation is well posed. The inverse transformation from the Jordan frame back to the Einstein frame is not possible for all field values in all theories, but we obtain a fully invertible transformation for vector-tensor theories by a redefinition of the vector field. Our main motivation is a better understanding of spontaneous scalarization and its generalizations, however our conclusions are applicable to a wide class of theories. Jordan frame has been traditionally used for certain calculations in scalar-tensor theories of gravitation, and our results will help researchers generalize these results, enabling comparison to observational data.
\end{abstract}

\section{INTRODUCTION}

Scalar-tensor theories (STTs) have been among the most popular alternatives to general relativity (GR), and also had a large impact on cosmology [1]. These theories commonly posit that gravitation is governed by scalar degrees of freedom in addition to the usual metric of general relativity, but their phenomenology can be very diverse otherwise due to various different coupling terms in their actions. An important feature of STTs is the freedom to choose the fundamental field variables while formulating them, e.g. one is always free to redefine a new metric $\tilde{g}_{\mu \nu}$ by scaling a given metric $g_{\mu \nu}$ by a function of the scalar $\phi: \tilde{g}_{\mu \nu}=A^{2}(\phi) g_{\mu \nu}$. Possibilities in such redefinitions are infinite, but two specific cases, called frames, have been of special importance. The first is the Jordan frame where the fundamental metric field of the theory couples minimally to matter degrees of freedom, and the second is the Einstein frame where the metric is such that the metric action is in the Einstein-Hilbert form, hence identical to that of GR [2].

Einstein and Jordan frames have been investigated in great detail in the literature which has shown their equivalence in many cases [2, revealed that one frame can be more useful for analyzing specific problems such as approximation schemes [1, 3 15, and even led to the discovery of previously overlooked STTs [6]. The aim of this work is generalizing the analysis of the relationship between these two frames to theories that contain higher spin fields such as vectors instead of scalars, or less common conformal scaling functions $A(\phi)$ such as those that depend on field derivatives.

Our main motivation is the recently investigated phenomenon of spontaneous tensorization which is a generalization of spontaneous scalarization in the scalar tensor theories introduced by Damour and Esposito-Farèse (DEF) [7. In DEF theories, the scalar fields spontaneously grow to large values from arbitrarily small perturbations near neutron stars due to a tachyonic instability. Such a theory, with some minor caveats, confirms to all known weak-field tests while providing large deviations from GR in the strong field, hence provides an especially good target to be tested using gravitational wave observations [8, 9. The desirable controlled spontaneous growth in DEF theories is not a direct results of the scalar nature of the coupling, or the tachyonic nature of the instability. Any field that carries an instability, such as a ghost on a vector field, in principle can lead to similar spontaneous growth which is called spontaneous tensorization [10, 11]. Overall, the theory of DEF is but one member of a large family of theories with similar observational signatures, all of which can be potentially tested with gravitational waves in the near future [11.

All spontaneous tensorization theories have been formulated in the Einstein frame for reasons we will discuss, and he main theme of this study is their properties in the Jordan frame. Despite our motivation, we will not specify our coupling terms to those that incite spontaneous growth, hence our results are general. We will use the terminology of Einstein and Jordan frames in a generalized sense, the former is always the one where the gravitational action is in the Einstein-Hilbert form, and the latter is always the one where matter fields couple to the metric minimally.

In Sec. II we present the tranformation between Einstein and Jordan frames in the quintessential STT of Brans and Dicke (BD) [12] whose conformal matter coupling structure is kept in all other theories we are interested. In Sec. III we obtain the Jordan frame for a vectortensor theory. In Sec. IV we go back to scalar fields, but this time study derivative couplings. We demonstrate the existence of higher derivative terms in the Jordan frame, commonly indicative of ill-posedness, and present the results from the existing literature which resolve this problem. We also discuss the invertibility of the frame transformations. In Sec. V we analyze the most general spontaneous tensorization theory which also has potentially dangerous higher derivative terms in the Jordan frame. We address this problem by showing that the equations of motion have at most second order time derivatives. In 
the last section, we summarize and discuss our results.

\section{CHANGING THE FRAME IN SCALAR-TENSOR THEORIES}

The most elementary case to compare the Einstein and Jordan frames is the Brans Dicke theory which was first introduced in the Jordan frame 12

$$
\begin{aligned}
& \frac{1}{16 \pi} \int d^{4} x \sqrt{-\tilde{g}} \Phi \tilde{R}-\frac{1}{16 \pi} \int d^{4} x \sqrt{-\tilde{g}} \frac{\omega(\Phi)}{\Phi} \tilde{g}^{\mu \nu} \partial_{\mu} \Phi \partial_{\nu} \Phi \\
& \quad+S_{m}\left[f_{m}, \tilde{g}_{\mu \nu}\right]
\end{aligned}
$$

where $\Phi$ is the scalar field, $\tilde{R}$ is the Ricci scalar associated with the Jordan frame metric $\tilde{g}_{\mu \nu}$, and $f_{m}$ denotes any matter degrees of freedom in the spacetime with their respective action $S_{M} . \omega=$ constant for $\mathrm{BD}$, but it can be generalized to obtain other STTs, for example $\omega=$ $-3 / 2-1 /(2 \beta \log \Phi)$ with a negative constant $\beta$ gives the spontaneous scalarization of DEF [5].

Eq. 1 deviates from the action of a scalar field minimally coupled to GR by the $\Phi$ factor in the EinsteinHilbert-like first term, and by the non-canonical scalar field action. The former issue can be addressed by expressing the action in terms of another metric which is conformally related the the original, $g_{\mu \nu}=A^{-2} \tilde{g}_{\mu \nu}$, since then

$$
\begin{aligned}
\tilde{R} & =A^{-2} R-6 g^{\mu \nu} A^{-3} \nabla_{\mu} \nabla_{\nu} A \\
\Leftrightarrow R & =A^{2} \tilde{R}+6 \tilde{g}^{\mu \nu} A^{-1} \tilde{\nabla}_{\mu} \tilde{\nabla}_{\nu} A
\end{aligned}
$$

which can be shown by straightforward calculation of the Ricci scalar in 4 spacetime dimensions. Here, all quantities with a tilde are related to $\tilde{g}_{\mu \nu}$, and bare ones are related to $g_{\mu \nu} \cdot{ }^{1}$ Remembering that $\tilde{g}=A^{4} g$ and $\tilde{g}^{\mu \nu}=A^{-2} g^{\mu \nu}$, we immediately see that the choice $\tilde{g}_{\mu \nu}=\Phi^{-1} g_{\mu \nu}$, puts the action in the form

$$
\begin{aligned}
& \frac{1}{16 \pi} \int d^{4} x \sqrt{-g}\left[R-\frac{3 / 2}{\Phi^{2}} g^{\mu \nu} \partial_{\mu} \Phi \partial_{\nu} \Phi\right] \\
& -\frac{1}{16 \pi} \int d^{4} x \sqrt{-g} \frac{\omega(\Phi)}{\Phi^{2}} g^{\mu \nu} \partial_{\mu} \Phi \partial_{\nu} \Phi \\
& \quad+S_{m}\left[f_{m}, \Phi^{-1} g_{\mu \nu}\right]
\end{aligned}
$$

up to boundary terms. The first term is exactly that of GR as we desired.

The second problem of having a non-canonical scalar action can also be addressed by using our freedom to redefine the scalar field. Introducing $\phi$ such that

$$
\frac{d \Phi}{d \phi}=\sqrt{\frac{4 \Phi^{2}}{3+2 \omega(\Phi)}}
$$

\footnotetext{
1 Our tilde convention follows DEF and the spontaneous scalarization/tensorization literature. The opposite is sometimes employed e.g. in 6].
}

finally puts the action into the commonly used Einstein frame form

$$
\begin{aligned}
& \frac{1}{16 \pi} \int d^{4} x \sqrt{-g} R-\frac{1}{16 \pi} \int d^{4} x \sqrt{-g} 2 g^{\mu \nu} \partial_{\mu} \phi \partial_{\nu} \phi \\
& \quad+S_{m}\left[f_{m}, A^{2}(\phi) g_{\mu \nu}\right] .
\end{aligned}
$$

We call $A(\phi) \equiv(\Phi(\phi))^{-1 / 2}$ the conformal scaling function. All the "alternative" nature of this action is in its matter coupling, the first two terms simply represent a scalar field living under GR. The price to have these familiar action terms is the nonminimal matter coupling. For the BD and DEF theories, the scalar field redefinitions are

$$
\Phi=\exp \left(\sqrt{\frac{4}{3+2 \omega}} \phi\right), \Phi=\exp \left(-\beta \phi^{2}\right)
$$

respectively.

\section{JORDAN FRAME IN THEORIES OF SPONTANEOUS VECTORIZATION}

A close examination of the DEF theory in Eq. 5 shows that one can get similar alternative theories of gravity by replacing the scalar by a vector as 10,13 .

$$
\begin{aligned}
& \frac{1}{16 \pi} \int d^{4} x \sqrt{-g} R-\frac{1}{16 \pi} \int d^{4} x \sqrt{-g} g^{\mu \rho} g^{\nu \sigma} F_{\rho \sigma} F_{\mu \nu} \\
& \quad+S_{m}\left[f_{m}, A_{X}^{2}(x) g_{\mu \nu}\right], x=g^{\mu \nu} X_{\mu} X_{\nu},
\end{aligned}
$$

where $X_{\mu}$ is a vector field that governs gravity in addition to the metric, similarly to the scalar field in STTs, and $F_{\mu \nu}=\nabla_{\mu} X_{\nu}-\nabla_{\nu} X_{\mu}=\partial_{\mu} X_{\nu}-\partial_{\nu} X_{\mu}$. We keep all vector-related quantities in the lower index to explicitly see the the inverse metric terms.

It is clear from this presentation why Einstein frame is more suitable to generalize STTs to vectors or other fields. Both the metric (Einstein-Hilbert) and the scalar field actions are in their "standard" forms, hence one keeps the Einstein-Hilbert action and replaces the action of the scalar with the standard action for a vector field to obtain a "vector-tensor" theory. ${ }^{2}$ Whereas, it is hard to see how to change the unusual scalar field terms in the Jordan frame of Eq. 1 to those of vectors. In Eq. 7 , modification to GR comes in the form of a confomal scaling of the metric that interacts with matter fields, just as for the DEF or BD theories. Then our only choice is the function $A_{X}$. It has been shown that $A_{X}=e^{\beta_{X} x / 2}$ with a constant $\beta_{X}$ or a similar function whose leading behavior around 0 is second order in its argument $x$ leads

\footnotetext{
${ }^{2}$ In the original formulation the vector is massive to conform to some possible observational bounds [10. However, intrinsic mass of vectors or scalars do not affect the current discussion.
} 
to spontaneously growing vector fields in the vicinity of neutron stars in analogy to spontaneous scalarization.

Remembering that we define the Jordan frame of a theory as where the metric couples minimally to the matter fields, we want to express the action in terms of $\tilde{g}_{\mu \nu}=A_{X}^{2} g_{\mu \nu}$ for which the action becomes

$$
\begin{aligned}
& \quad \frac{1}{16 \pi} \int d^{4} x \sqrt{-\tilde{g}} \bar{A}_{X}^{-2} \tilde{R}-\frac{1}{16 \pi} \int d^{4} x \sqrt{-\tilde{g}} \tilde{g}^{\mu \rho} \tilde{g}^{\nu \sigma} F_{\rho \sigma} F_{\mu \nu} \\
& +\frac{6}{16 \pi} \int d^{4} x \sqrt{-\tilde{g}} \tilde{g}^{\mu \nu} \partial_{\mu} \bar{A}_{X}^{-1} \partial_{\nu} \bar{A}_{X}^{-1} \\
& \quad+S_{m}\left[f_{m}, \tilde{g}_{\mu \nu}\right] .
\end{aligned}
$$

$\bar{A}_{X}$ is simply $A_{X}$, but now implicitly defined as a function of the field variables in the new frame by

$$
\bar{A}_{X}\left(\tilde{g}^{\mu \nu} X_{\mu} X_{\nu}\right)=A_{X}\left(\bar{A}_{X}^{2} \tilde{g}^{\mu \nu} X_{\mu} X_{\nu}\right)
$$

For example, the exponential function we used before now means

$$
\bar{A}_{X}^{2}=e^{\beta_{X} \tilde{g}^{\mu \nu} X_{\mu} X_{\nu} \bar{A}_{X}^{2}}
$$

The implicit definition in Eq. 9 is unappealing, and more importantly cannot be always inverted for given $\left\{\tilde{g}_{\mu \nu}, X_{\mu}\right\}$, rendering the theory meaningless for such values in the Jordan frame. We will discuss this issue in more detail in the coming sections, but we can address it for this specific theory by utilizing the freedom to redefine the vector field. Consider

$$
\tilde{X}_{\mu} \equiv \bar{A}_{X} X_{\mu}
$$

Now we can re-express $A_{X}$ as a function of $\tilde{g}_{\mu \nu}$ and $\tilde{X}_{\mu}$ as

$$
\tilde{A}_{X}\left(\tilde{g}^{\mu \nu} \tilde{X}_{\mu} \tilde{X}_{\nu}\right)=A_{X}\left(\tilde{g}^{\mu \nu} \tilde{X}_{\mu} \tilde{X}_{\nu}\right)
$$

that is $\tilde{A}_{X}$ has the exact same functional form as $A_{X}$, but for a different set of variables. For the exponential function we considered before

$$
\tilde{A}_{X}=e^{\beta_{X} \tilde{g}^{\mu \nu} \tilde{X}_{\mu} \tilde{X}_{\nu} / 2} .
$$

Thus, one can transform from one frame and set of field variables to the other in the straightforward manner

$$
\begin{aligned}
\tilde{g}_{\mu \nu} & =A_{X}^{2} g_{\mu \nu} & g_{\mu \nu} & =\tilde{A}_{X}^{-2} \tilde{g}_{\mu \nu} \\
\tilde{X}_{\mu} & =A_{X} X_{\mu} & X_{\mu} & =\tilde{A}_{X}^{-1} \tilde{X}_{\mu} \\
A_{X} & =e^{\beta_{X} g^{\mu \nu} X_{\mu} X_{\nu} / 2} & \tilde{A}_{X} & =e^{\beta_{X} \tilde{g}^{\mu \nu} \tilde{X}_{\mu} \tilde{X}_{\nu} / 2}
\end{aligned}
$$

The transformation $\left\{g_{\mu \nu}, X_{\mu}\right\} \leftrightarrow\left\{\tilde{g}_{\mu \nu}, \tilde{X}_{\mu}\right\}$ is invertible. We will see that this is not always the case in other generalizations of STTs.

Finally, the Jordan frame action in terms of the new variables $\left\{\tilde{g}_{\mu \nu}, \tilde{X}_{\mu}\right\}$ is given by

$$
\begin{aligned}
& \frac{1}{16 \pi} \int d^{4} x \sqrt{-\tilde{g}} \tilde{A}_{X}^{-2} \tilde{R}_{-} \frac{1}{16 \pi} \int d^{4} x \sqrt{-\tilde{g}} \tilde{A}_{X}^{-2} \tilde{F}^{\mu \nu} \tilde{F}_{\mu \nu} \\
& -\frac{2}{16 \pi} \int d^{4} x \sqrt{-\tilde{g}} \tilde{A}_{X}^{-2} \tilde{\Lambda}_{X}^{2}\left[\left(\tilde{x}-3-\tilde{\Lambda}_{X}^{-1}\right) \tilde{\nabla}^{\mu} \tilde{x}_{\bar{\nabla}} \tilde{\nabla}^{\tilde{x}}\right. \\
& \left.\quad-\left(\tilde{X}^{\mu} \tilde{\nabla}_{\mu} \tilde{x}\right)^{2}+2 \tilde{\Lambda}_{X}^{-1}\left(\tilde{X}^{\rho} \tilde{\nabla}_{\rho} \tilde{X}^{\mu}\right) \tilde{\nabla}_{\mu} \tilde{x}\right] \\
& +S_{m}\left[f_{m}, \tilde{g}_{\mu \nu}\right],
\end{aligned}
$$

where $\tilde{F}_{\mu \nu} \equiv \tilde{\nabla}_{\mu} \tilde{X}_{\nu}-\tilde{\nabla}_{\nu} \tilde{X}_{\mu}, \tilde{x} \equiv \tilde{g}^{\mu \nu} \tilde{X}_{\mu} \tilde{X}_{\nu}, \tilde{\Lambda}_{X} \equiv$ $\tilde{A}_{X}^{-1}\left(d \tilde{A}_{X} / d \tilde{x}\right)$ and all raising is performed with $\tilde{g}^{\mu \nu}$. The action for the vector field is not the standard one, but this is expected in the Jordan frame as in Eq. 1.

We should note that the actions in Eq. 8 and Eq. 15 both satisfy our definition of the Jordan frame since they both have minimal matter coupling. However, we will prefer the latter since it does not suffer from the nonexistence of $\bar{A}_{X}$ for certain field values in Eqs. 9 , 10, hence we consider $\left\{\tilde{g}_{\mu \nu}, \tilde{X}_{\mu}\right\}$ to provide a more natural setting for the Jordan frame.

$\tilde{X}$ carries three degrees of freedom rather than the usual two in Maxwell fields since it lacks the gauge free$\operatorname{dom} \tilde{X}_{\mu} \rightarrow \tilde{X}_{\mu}+\partial_{\mu} \lambda$. We can consider the degree of freedom in the norm $\tilde{x}$ separately, and view $\tilde{X}_{\mu}$ as carrying the remaining two. Thus, we can treat $\tilde{x}$ as an independent scalar which couples to the vector action $\tilde{F}^{\mu \nu} \tilde{F}_{\mu \nu}$ in addition to the gravity term $\tilde{R}$. This is reminiscent of Einstein-Maxwell-scalar theories, especially the ones that feature a newly discovered type of spontaneous scalarization [14. This might lead to an alternative understanding of the vectorization process through the scalar degree of freedom in $\tilde{x}$, and provide new connections between spontaneous vectorization and spontaneous scalarization. Note that one can also define $\tilde{\chi}=\tilde{A}_{X}^{-2}$ and express Eq. 15 in somewhat closer resemblance to Eq. 1 where $\tilde{\chi}$ would behave like a scalar.

\section{JORDAN FRAME FOR GHOST-BASED SPONTANEOUS SCALARIZATION}

A second avenue to generalize spontaneous growth of DEF is using a different instability, as opposed to using a different field. More concretely, the tachyonic can be replaced by a ghost if the conformal scaling depends on the derivative terms in the action

$$
\begin{aligned}
& \frac{1}{16 \pi} \int d^{4} x \sqrt{-g} R-\frac{1}{16 \pi} \int d^{4} x \sqrt{-g} 2 g^{\mu \nu} \partial_{\mu} \phi \partial_{\nu} \phi \\
& \quad+S_{m}\left[f_{m}, A_{\partial}^{2}(K) g_{\mu \nu}\right], K \equiv g^{\mu \nu} \partial_{\mu} \phi \partial_{\nu} \phi .
\end{aligned}
$$

The resulting theory leads to scalarization of neutron stars, e.g for the choice $A_{\partial}(K)=e^{\beta_{\partial} K / 2}$ with some constant $\beta_{\partial}$ [1]. This theory is named ghost-based spontaneous scalarization since it can be shown that small perturbations around the scalar field vacuum behave like 
a ghost, but this instability is suppressed as the field grows.

Using Eq. 2, we can transform to the Jordan frame with $\tilde{g}_{\mu \nu}=A_{\partial}^{2} g_{\mu \nu}$

$$
\begin{aligned}
& \frac{1}{16 \pi} \int d^{4} x \sqrt{-\tilde{g}} \tilde{A}_{\partial}^{-2} \tilde{R}-\frac{2}{16 \pi} \int d^{4} x \sqrt{-\tilde{g}} \tilde{A}_{\partial}^{-2} \tilde{K} \\
& +\frac{6}{16 \pi} \int d^{4} x \sqrt{-\tilde{g}} \tilde{g}^{\mu \nu} \partial_{\mu} \tilde{A}_{\partial}^{-1} \partial_{\nu} \tilde{A}_{\partial}^{-1} \\
& \quad+S_{m}\left[f_{m}, \tilde{g}_{\mu \nu}\right], \tilde{K} \equiv \tilde{g}^{\mu \nu} \partial_{\mu} \phi \partial_{\nu} \phi .
\end{aligned}
$$

$\tilde{A}_{\partial}(\tilde{K})$ is defined implicitly through

$$
\tilde{A}_{\partial}(\tilde{K})=A_{\partial}\left(\tilde{A}_{\partial}^{2}(\tilde{K}) \tilde{K}\right)
$$

e.g. the exponential conformal scaling above leads to ${ }^{3}$

$$
\tilde{A}_{\partial}^{2}=e^{\beta_{\partial} \tilde{K} \tilde{A}_{\partial}^{2}} .
$$

Even though this Jordan frame formulation looks quite similar to that of the vector field case in Eq. 8 and 9 , it brings in new issues to tackle. First, Eq. 17 contains terms with more than one time derivatives of $\phi$ through $\partial_{\mu} \tilde{A}_{\partial}$, which might indicate an unphysical nature due to Ostrogradsky's theorem [15. This is peculiar, since we do not expect the nature of the theory to change radically from one frame to the other, and there are no higher time derivative terms in the Einstein frame formulation in Eq. 16 .

This puzzle can be resolved by considering the equations of motion in the Jordan frame $[\underline{6}$

$$
\begin{aligned}
\tilde{G}_{\mu \nu} & =8 \pi \tilde{A}_{\partial}^{2} \tilde{T}_{\mu \nu}-2 \tilde{A}_{\partial}\left(\tilde{g}_{\mu \nu} \tilde{\square}^{-1}-\tilde{\nabla}_{\mu} \tilde{\nabla}_{\nu} \tilde{A}_{\partial}^{-1}\right) \\
& -2 \tilde{\Lambda}_{\partial}\left(6 \tilde{A}_{\partial} \tilde{\square} \tilde{A}_{\partial}^{-1}-\tilde{R}\right) \partial_{\mu} \phi \partial_{\nu} \phi \\
& +\tilde{A}_{\partial}^{-2} \tilde{g}_{\mu \nu} \tilde{g}^{\rho \sigma} \partial_{\sigma} \tilde{A}_{\partial} \partial_{\rho} \tilde{A}_{\partial}-4 \tilde{A}_{\partial}^{-2} \partial_{\mu} \tilde{A}_{\partial} \partial_{\nu} \tilde{A}_{\partial} \\
& +2 \partial_{\mu} \phi \partial_{\nu} \phi-\tilde{g}_{\mu \nu} \tilde{K}-4 \tilde{\Lambda}_{\partial} \tilde{K} \partial_{\mu} \phi \partial_{\nu} \phi \\
0 & =\tilde{\nabla}_{\mu}\left(\tilde{\Lambda}_{\partial} \tilde{A}_{\partial}^{-2}\left(6 \tilde{A}_{\partial} \tilde{\square} \tilde{A}_{\partial}^{-1}-\tilde{R}+2 \tilde{K}-\tilde{\Lambda}_{\partial}^{-1}\right) \tilde{\nabla}^{\mu} \phi\right)
\end{aligned}
$$

where $\tilde{\Lambda}_{\partial} \equiv \tilde{A}_{\partial}^{-1}\left(d \tilde{A}_{\partial} / d \tilde{K}\right)$. These equations indeed have up to fourth order time derivatives indicative of ill-posedness. However, the trace of the first equation gives

$$
6 \tilde{A}_{\partial} \tilde{\square} \tilde{A}_{\partial}^{-1}-\tilde{R}=\frac{8 \pi \tilde{A}_{\partial}^{2} \tilde{T}-2 \tilde{K}-4 \tilde{\Lambda}_{\partial} \tilde{K}^{2}}{1+2 \tilde{\Lambda}_{\partial} \tilde{K}} .
$$

Hence, as long as the stress energy tensor of matter is first order, $6 \tilde{A}_{\partial} \tilde{\square} \tilde{A}_{\partial}^{-1}-\tilde{R}$ can be re-expressed in terms of first derivatives, and $\partial_{t}^{2} \tilde{A}_{\partial}$ can be re-expressed in terms

3 We can have an explicit definition in this specific case in terms of the relatively well-known Lambert $W$ function $W\left(x e^{x}\right) \equiv x$ : $\tilde{A}_{\partial}(\tilde{K})=\sqrt{W\left(-\beta_{\partial} \tilde{K}\right) /\left(-\beta_{\partial} \tilde{K}\right)}$. However, the definition is implicit for generic $A_{\partial}$. of at most second time derivatives of $\phi$ and $\tilde{g}_{\mu \nu}$. Inserting these identities back ensures that Eq. 20 contains at most second time derivatives [6].

The second issue in the Jordan frame formulation is the implicit definition of $\tilde{A}_{\partial}$ in Eq. 18 which does not necessarily have a solution for all $\tilde{K}$ for a given $A_{\partial}$. For example, the exponential function we discussed (Eq. 19) only provides a solution for $\tilde{A}_{\partial}$ if $\beta_{\partial} \tilde{K}<e^{-1}$ and is multi-valued for positive $\beta_{\partial} \tilde{K}$. This also implies that it is not always possible to invert $\tilde{g}_{\mu \nu}$ to $g_{\mu \nu}$. In some sense, all possible values of $\left\{\tilde{g}_{\mu \nu}, \phi\right\}$ is too big a configuration space for our theory. This is not the case for all $\tilde{A}_{\partial}$, for example the choice $A_{\partial}(K)=e^{\gamma_{\partial} K^{2}}$ leads to a $\tilde{A}_{\partial}(\tilde{K})$ defined for all $\tilde{K}$ values $[\underline{6}$.

Non-invertibility of the frame transformation is not a new problem, and is present in the simplest STTs such as BD as well. In Eq. 1, $\Phi$ is constrained to be positive. Field variables cannot be transformed to the Jordan frame otherwise due to Eq. 6, and the action also becomes meaningless for the DEF theory due to the logarithmic terms in $\omega(\Phi)$. Nevertheless, we would still want to have an invertible transformation between frames for as much of the configuration space as possible.

Note that we encountered the non-invertibility problem in vector-tensor theories as well (Eq. 9, 10), but the redefinition of the vector field, $X_{\mu} \rightarrow \tilde{X}_{\mu}$ resolved the issue by providing a fully invertible transformation between the frames in Eq. 14. In other words, the full range of $\tilde{X}_{\mu}$ was just right for our theory while that of $X_{\mu}$ was "too big". We were not able to construct any such redefinition $\phi \rightarrow \tilde{\phi}$ in the current case. As an example, the most obvious candidate

$$
\partial_{\mu} \tilde{\phi} \equiv \tilde{A}_{\partial} \partial_{\mu} \phi
$$

leads to the explicit definition $\tilde{A}_{\partial}=A_{\partial}\left(\tilde{g}^{\mu \nu} \partial_{\mu} \tilde{\phi} \partial_{\nu} \tilde{\phi}\right)$ and a fully invertible tranformation between frames. However, this also implies $\partial_{\mu} \partial_{\nu} \tilde{\phi} \neq \partial_{\nu} \partial_{\mu} \tilde{\phi}$, hence such a differentiable $\tilde{\phi}$ does not exist.

A related problem to the nonexistence of $\tilde{A}_{\partial}$ for certain field values would be the following: if we start with some initial data $\left\{\tilde{g}_{\mu \nu}, \phi\right\}$ and their first derivatives in the Jordan frame for which $\tilde{A}_{\partial}$ is defined, does the time evolution lead to values of $\left\{\tilde{g}_{\mu \nu}, \phi\right\}$ for which $\tilde{A}_{\partial}$ is defined everywhere in the future of the initial data? Since the non-existence of $A_{\partial}$ is not a problem in the Einstein frame for any values of $\left\{g_{\mu \nu}, \phi\right\}$, we would expect the answer to be affirmative. A proper analysis requires tools from the theory of partial differential equations, and we hope mathematical physics can provide some insight for this problem which has not been addressed in the gravitational physics literature to the best of our knowledge.

Lastly, the fact that the equations of motion are ultimately second order in time derivatives in the Jordan frame may suggests that there is also a field redefinition that would allow the action to contain only first order derivatives, but we could not identify a simple example 
of this aside from the trivial transformation of going back to the Einstein frame.

\section{JORDAN FRAME FOR GENERIC SPONTANEOUS TENSORIZATION}

We have seen that spontaneous growth can be generalized from scalars to vectors, or from a tachyonbased mechanism to a ghost-based mechanism. This approach can be continued to various other fields such as spinors [16, other mechanisms such as spontaneous growth through the Higgs mechanism [17, or any combination of them. All these form the family of spontaneous tensorization theories.

How generic are the issues of higher time derivatives and invertibility of frame transformations that we encountered in Sec. IV? If they appear in a generic spontaneous tensorization theory, can they be resolved similarly to the case of ghost-based spontaneous scalarization? Consider the following general action for which all the theories we have investigated so far are special cases

$$
\begin{aligned}
& \frac{1}{16 \pi} \int d^{4} x \sqrt{-g} R-\frac{1}{16 \pi} \int d^{4} x \sqrt{-g} \mathcal{L}_{\psi}\left(g^{\mu \nu}, \psi, \partial \psi\right) \\
& \quad+S_{m}\left[f_{m}, A_{\psi}^{2}\left(g^{\mu \nu}, \psi, \partial \psi\right) g_{\mu \nu}\right] .
\end{aligned}
$$

Here, $\psi$ is any field, potentially with multiple tensor indices, and $\partial \psi$ is a collective symbol for its first derivatives. The Jordan frame of such a theory is obtained by $\tilde{g}_{\mu \nu}=A_{\psi}^{2} g_{\mu \nu}$ which has the action

$$
\begin{aligned}
& \quad \frac{1}{16 \pi} \int d^{4} x \sqrt{-\tilde{g}} \tilde{A}_{\psi}^{-2} \tilde{R}-\frac{1}{16 \pi} \int d^{4} x \sqrt{-\tilde{g}} \tilde{\mathcal{L}}_{\psi}\left(\tilde{g}^{\mu \nu}, \psi, \partial \psi\right) \\
& +\frac{6}{16 \pi} \int d^{4} x \sqrt{-\tilde{g}} \tilde{g}^{\mu \nu} \partial_{\mu} \tilde{A}_{\psi}^{-1} \partial_{\nu} \tilde{A}_{\psi}^{-1} \\
& \quad+S_{m}\left[f_{m}, \tilde{g}_{\mu \nu}\right] .
\end{aligned}
$$

where $\tilde{A}_{\psi}\left(\tilde{g}^{\mu \nu}, \psi, \partial \psi\right)$ is defined implicitly as

$$
\tilde{A}_{\psi}=A_{\psi}\left(\tilde{A}_{\psi}^{2} \tilde{g}^{\mu \nu}, \psi, \partial \psi\right)
$$

and

$$
\tilde{\mathcal{L}}_{\psi}\left(\tilde{g}^{\mu \nu}, \psi, \partial \psi\right)=\tilde{A}_{\psi}^{-4} \mathcal{L}_{\psi}\left(\tilde{A}_{\psi}^{2} \tilde{g}^{\mu \nu}, \psi, \partial \psi\right)
$$

The crucial point is that, $\tilde{\mathcal{L}}_{\psi}$ and $\tilde{A}_{\psi}$ are still only functions of at most the first derivatives of $\psi$.
The equations of motion in the Jordan frame are

$$
\begin{aligned}
\tilde{G}_{\mu \nu} & =8 \pi \tilde{A}_{\psi}^{2} \tilde{T}_{\mu \nu}-2 \tilde{A}_{\psi}\left(\tilde{g}_{\mu \nu} \tilde{\square}_{\psi}^{-1}-\tilde{\nabla}_{\mu} \tilde{\nabla}_{\nu} \tilde{A}_{\psi}^{-1}\right) \\
& -2\left(6 \tilde{A}_{\psi} \tilde{\square}_{\psi}^{-1}-\tilde{R}\right) \tilde{A}_{\psi}^{-1} \frac{\delta \tilde{A}_{\psi}}{\delta \tilde{g}^{\mu \nu}} \\
& +\tilde{A}_{\psi}^{-2} \tilde{g}_{\mu \nu} \tilde{g}^{\rho \sigma} \partial_{\sigma} \tilde{A}_{\psi} \partial_{\rho} \tilde{A}_{\psi}-4 \tilde{A}_{\psi}^{-2} \partial_{\mu} \tilde{A}_{\psi} \partial_{\nu} \tilde{A}_{\psi} \\
& -\frac{\tilde{A}_{\psi}^{2}}{\sqrt{-\tilde{g}}} \frac{\delta\left(\sqrt{-\tilde{g}} \tilde{\mathcal{L}}_{\psi}\right)}{\delta \tilde{g}^{\mu \nu}} \\
0 & =\frac{1}{\sqrt{-\tilde{g}}} \partial_{\mu}\left(\sqrt{-\tilde{g}} \tilde{A}_{\psi}^{-3}\left(6 \tilde{A}_{\psi} \tilde{\square}_{\psi}^{-1}-\tilde{R}\right) \frac{\delta \tilde{A}_{\psi}}{\delta\left(\partial_{\mu} \psi\right)}\right) \\
& -\tilde{A}_{\psi}^{-3}\left(6 \tilde{A}_{\psi} \tilde{\square} \tilde{A}_{\psi}^{-1}-\tilde{R}\right) \frac{\delta \tilde{A}_{\psi}}{\delta \psi}-\frac{1}{2} \frac{\delta \tilde{\mathcal{L}}_{\psi}}{\delta \psi} .
\end{aligned}
$$

The second equation contains the fourth derivative term $\tilde{\nabla}_{\mu} \tilde{\square} \tilde{A}_{\psi}^{-1}$ and both equations have third time derivatives of $\psi$. Both cases are potentially problematic. However, the trace of the first equation implies

$$
6 \tilde{A}_{\psi} \tilde{\square} \tilde{A}_{\psi}^{-1}-\tilde{R}=\frac{8 \pi \tilde{T}-\frac{\tilde{A}_{\psi}^{2}}{\sqrt{-\tilde{g}}} \frac{\delta\left(\sqrt{-\tilde{g}} \tilde{\mathcal{L}}_{\psi}\right)}{\delta \tilde{g}^{\mu \nu}}}{1+2 \tilde{A}_{\psi}^{-1} \frac{\delta \tilde{A}_{\psi}}{\delta \tilde{g}^{\mu \nu}}},
$$

in complete analogy to Eq. 21. $6 \tilde{A}_{\psi} \tilde{\square} \tilde{A}_{\psi}^{-1}-\tilde{R}$ can be expressed in terms of at most first derivatives of the fundamental field variables $\left\{\tilde{g}_{\mu \nu}, \psi\right\}$ as long as $\tilde{A}_{\psi}, \tilde{\mathcal{L}}_{\psi}$ and matter stress-energy contain at most first derivatives. This in turn means $\partial_{t}^{2} \tilde{A}_{\psi}$ can also be expressed in terms of at most two time derivatives. Hence, changing from the Einstein frame (Eq. 23), to the Jordan frame (Eq.24) does not introduce ill-posedness.

Note that our starting action Eq. 23 in the Einstein frame is general enough to contain potential self interaction terms for $\psi$ in $\mathcal{L}$, and a conformal scaling $A_{\psi}$ that depends both on $\psi$ and its derivatives. Thus, our results continue to hold for the spontaneous growth of massive scalars or vectors, or theories where ghost-based and tachyon-based instabilities are present at the same time. It is also trivial to generalize the result to a collection if fields $\psi^{(i)}$. These cover all examples of spontaneous tensorization in the literature [10, 11, 16, 17]. All these theories, aside from spontaneous vectorization in Sec. III] also contain conformal scaling functions which contain derivatives. Hence, the resolution of the higher time derivative problem we outlined is central to their viability in the Jordan frame as physical theories.

Invertibility of the frame transformation in relation to the existence of a solution to Eq. 26 for $\tilde{A}_{\psi}$ is not resolved in general. All possible values of $\psi$ provide too big a configuration space in the Jordan frame, and the questions we posed in Sec. IV are open in this generic case as well. However, we remind that such problems are present even in the DEF theory. 


\section{CONCLUSIONS}

We studied the Jordan frame formulation of generalizations of STTs, where the scalar is replaced with other fields, and couplings can depend on derivatives. Our motivation came from the specific class of theories that feature spontaneous tensorization. These are most naturally defined in the Einstein frame where the action for the additional field to the metric (e.g. the vector) is in the canonical form. However, our results can be applied to any generalization of STTs that is based on a conformal scaling of the metric in the matter action by some function of a dynamical field and its first derivatives.

The first case we examined is the vector-tensor theory obtained by replacing the scalar in the DEF theory by a vector where the conformal scaling function $A_{X}$ depends on the norm of the vector field. A completely invertible transformation (Eq. 14) can be obtained if the vector field is redefined in the Jordan frame, $X_{\mu} \rightarrow \tilde{X}_{\mu}=A_{X} X_{\mu}$, as well as the metric. Moreover, interesting connections can be observed to the recently discovered spontaneous growth in Einstein-Maxwell-scalar theories [14].

Jordan frame of ghost-based spontaneous scalarization where the conformal scaling depends on the derivatives of the scalar field presents challenges. First, the Jordan frame contains higher derivative terms indicative of illposedness due to Ostrogradsky's theorem, which is odd since this is not an issue in the Einstein frame, and we would not expect the nature of the theory to change in such radical fashion due to a frame change. The equations of motion indeed contain up to fourth order time derivatives, but it can be shown that such terms cancel each other to render the equations second order in time. It is also curious to see that the conformal scaling function cannot be defined for all values of the field variables $\left\{\tilde{g}_{\mu \nu}, \phi\right\}$ which causes the transformation between frames to be non-invertible. We could not find a formulation where this problem is resolved, but noted that this is the case even in the DEF theory where the scalar field is restricted to be positive in the Jordan frame. It is important to understand the meaning of the field values in the Jordan frame where the transformation back to the
Einstein frame is not defined, which we leave to future studies.

We finally showed that a generic spontaneous tensorization theory contains higher time derivative terms in its formulation, much like ghost-based spontaneous scalarization. The equations of motion are again ultimately rendered second order in time, even though they naively contain fourth time derivatives, demonstrating that the Jordan frame formulation does not introduce illposedness. We should add at this point that the fact that there are only first derivative terms in the Einstein frame action does not guarantee well-posedness. A theory can be rendered unphysical by other factors such as indefinitely growing fields such as ghosts, even if the equations of motion have no more than two time derivatives. The Einstein frame formulation of spontaneous tensorization theories are not known to be completely free of such undesirable features [11, 16, 17, but our work here shows that transferring to the Jordan frame at least does not add new sources of ill-posedness.

Certain calculations on STTs have been performed using the Jordan frame such as the gravitational wave memory for the DEF theory 4]. Consequently, we believe this study will enable researchers to extend similar work to spontaneous tensorization in general. Possibility of nearfuture testing is a basic appeal of spontaneous scalarization and tensorization. Calculations of specific observational signs will enable the gravity community to compare the predictions of these theories to actual observations, and understand the differences between individual theories in the spontaneous tensorization family.

\section{Acknowledgments}

This project started with a question posed by Leonardo Gualtieri to whom we are grateful. The author is supported by Grant No. 117F295 of the Scientific and Technological Research Council of Turkey (TÜBITAK). We would like to acknowledge networking and travel support by the COST Action CA16104.
[1] Y. Fujii and K. Maeda, The scalar-tensor theory of gravitation (Cambridge University Press, 2007).

[2] E. E. Flanagan, Class. Quantum Grav. 21, 3817 (2004), arXiv:gr-qc/0403063.

[3] T. Damour and G. Esposito-Farese, Class.Quant.Grav. 9, 2093 (1992).

[4] S. M. Du and A. Nishizawa, Phys. Rev. D 94, 104063 (2016).

[5] E. Barausse, C. Palenzuela, M. Ponce, and L. Lehner, Phys. Rev. D 87, 081506 (2013), 1212.5053.

[6] M. Zumalacárregui and J. Garca-Bellido, Phys. Rev. D89, 064046 (2014), 1308.4685.

[7] T. Damour and G. Esposito-Farèse, Phys. Rev. Lett. 70,
2220 (1993).

[8] LIGO Scientific Collaboration and Virgo Collaboration, B. P. Abbott et al., Phys. Rev. Lett. 116, 061102 (2016).

[9] Virgo, LIGO Scientific Collaborations, B. P. Abbott et al., Phys. Rev. Lett. 119, 161101 (2017), 1710.05832.

[10] F. M. Ramazanoğlu, Phys. Rev. D96, 064009 (2017), 1706.01056.

[11] F. M. Ramazanoğlu, Phys. Rev. D97, 024008 (2018), 1710.00863.

[12] C. Brans and R. H. Dicke, Phys. Rev. 124, 925 (1961).

[13] J. Beltran Jimenez, A. L. Delvas Froes, and D. F. Mota, Phys. Lett. B725, 212 (2013), 1212.1923.

[14] C. A. R. Herdeiro, E. Radu, N. Sanchis-Gual, and J. A. 
Font, Phys. Rev. Lett. 121, 101102 (2018), 1806.05190.

[15] R. P. Woodard, Scholarpedia 10, 32243 (2015), revision \#186559.

[16] F. M. Ramazanoğlu, Phys. Rev. D98, 044011 (2018),
1804.00594.

[17] F. M. Ramazanoğlu, Phys. Rev. D98, 044013 (2018), 1804.03158. 